\title{
Bilateral Hypodontia of Mandibular Second Premolars and Lateral Incisors in a Nonsyndromic Patient: A Rare Case Report
}

\author{
${ }^{1}$ Neha Agarwal, ${ }^{2}$ Shefali Chaturvedy, ${ }^{3}$ Nikhil Marwah, ${ }^{4}$ Pooja Mishra, ${ }^{5}$ Shakun Kanjani
}

\begin{abstract}
Hypodontia is the term used to describe the developmental absence of one or more primary or permanent teeth, excluding the third molars. It is the most commonly occurring developmental dental anomaly and can be a challenge to manage clinically. Hypodontia can occur in association with syndrome or it may occur in nonsyndromic patient. Bilateral occurrence is common but it is very rare to see two bilateral congenital missing teeth in a nonsyndromic patient. This case report presents a rare occurrence of congenital bilateral missing mandibular second premolars and mandibular lateral incisors in a nonsyndromic patient.
\end{abstract}

Keywords: Bilateral, Hypodontia, Incisor-premolar.

How to cite this article: Agarwal N, Chaturvedy S, Marwah $\mathrm{N}$, Mishra P, Kanjani S. Bilateral Hypodontia of Mandibular Second Premolars and Lateral Incisors in a Nonsyndromic Patient: A Rare Case Report. J Mahatma Gandhi Univ Med Sci Tech 2016;1(1):24-26.

Source of support: Nil

Conflict of interest: None

\section{INTRODUCTION}

Congenital absence of one or more teeth is a common developmental anomaly that affect 3.2 to $7.6 \%$ of population. ${ }^{1}$ It involve complete or partial loss of teeth and presents a clinical dilemma whether to go for treating dentist. The problem lies not in common occurrence of congenital missing teeth but in selection of a treatment procedure that would give good results over a long span of time.

Stewart defined anodontia as complete absence of teeth, hypodontia as absence of one or few teeth and agenesis of numerous teeth as oligodontia (more than six). ${ }^{2}$ Disturbance during initiation and proliferation of tooth bud which is early stage of tooth formation process results in complete or partial loss of teeth. ${ }^{3}$

\footnotetext{
1,4,5 Postgraduate Student, ${ }^{2}$ Reader, ${ }^{3}$ Professor and Head ${ }^{1-5}$ Department of Pedodontics and Preventive Dentistry Mahatma Gandhi Dental College and Hospital, Mahatma Gandhi University of Medical Sciences and Technology, Jaipur Rajasthan, India.
}

Corresponding Author: Neha Agarwal, A-86, Manish Marg Nemi Nagar, Vaishali Nagar, Jaipur, Rajasthan, India, Phone: +91-8955398800, e-mail: dr.nehaag2011@gmail.com
Hypodontia can occur as a one of the clinical features of a syndromes or it may present itself as nonsyndromic. The nonsyndromic hypodontia can be either sporadic or familial with significant phenotypic variability which can be presented as autosomal-dominant, autosomalrecessive, or X-linked trait. Research has indicated that genetic and environmental factors play an important role in causing hypodontia. ${ }^{4}$

Hypodontia affects more commonly females as compared to males in the ratio of $3: 2 .^{5}$

It usually occurs in permanent dentition affecting mandibular second premolar (2.91-3.22\%) after third molar, followed by the maxillary lateral incisor (1.55$1.78 \%)$ and maxillary second premolar $(1.39-1.61 \%)^{2}$. It rarely affects deciduous dentition less than $1 \%$ of population and is usually associated with missing mandibular incisors. ${ }^{6}$

Although congenital bilateral missing teeth is a common occurrence, but it is rare to see bilateral two congenital missing teeth in mandibular arch of a nonsyndromic patient. This case report presents a rare occurrence of congenital bilateral missing mandibular second premolars and mandibular lateral incisors in a nonsyndromic patient.

\section{CASE REPORT}

A 9 years old male child reported to the OPD in the Department of Pedodontics and Preventive Dentistry, Mahatma Gandhi Dental College and Hospital, Jaipur, Rajasthan, accompanying his father with the chief complaint of pain in his lower right back tooth region since 20 days. A complete history of the patient was recorded. Family and medical history were noncontributory. General and extraoral examination did not reveal any abnormality.

Intraoral examination revealed bilaterally missing permanent lower lateral incisors and over-retained lower central incisor. Lower deciduous second molar on both the sides were observed to be grossly decayed and associated with localized buccal swelling (Fig. 1). Swelling was tender, fluctuant and around the neck of the tooth. Tenderness on percussion was present only in relation to right lower deciduous second molar. 


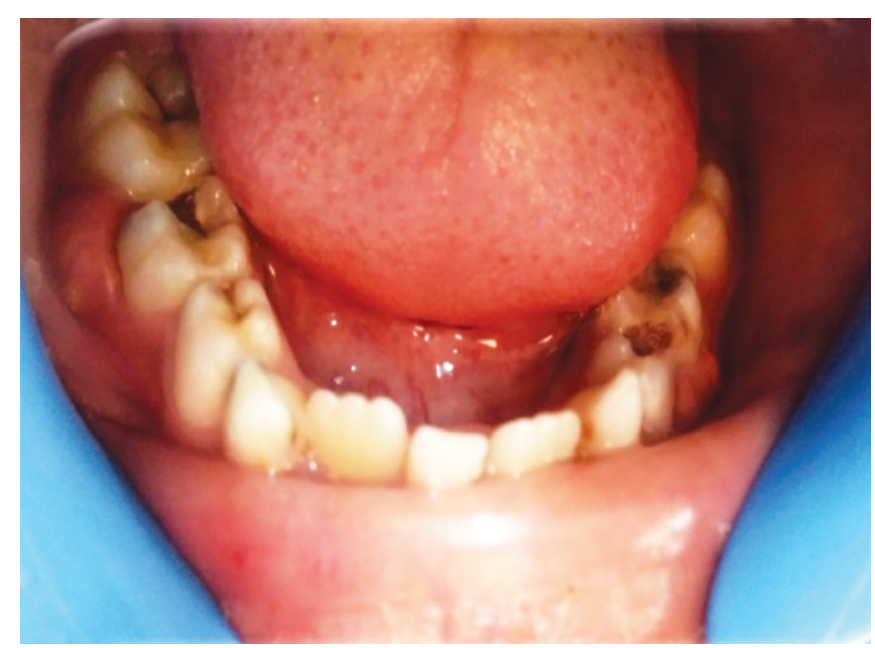

Fig. 1: Grossly decayed lower deciduous second molar with buccal swelling

Intraoral periapical radiolucency (IOPAR) of both sides revealed that the deciduous second molars had pulpal involvement with periapical radiolucency (Figs 2A and B). It was also observed that the erupting permanent first premolars were causing resorption of mesial roots of deciduous second molars. Orthopantomograph revealed congenitally missing bilateral mandibular lateral incisors and second premolars (Fig. 3). Treatment plan comprised of retaining mandibular deciduous second molars for as long as possible as natural space maintainer. To achieve this goal, lesion sterilization and tissue repair therapy with triple antibiotic paste were performed in the necrotic deciduous second molars on either side followed by extraction of mandibular deciduous canines and first molars bilaterally. These extractions were done to accelerate the eruption of permanent first premolars into their right space, thus preventing the resorption pressure on roots of deciduous second molars.

After a 3 weeks follow-up, buccal swelling had subsided. Also the permanent first premolars were erupting along the straight pathway of eruption indicating positive outcome from designed treatment plan (Figs 4A and B).

\section{DISCUSSION}

Tooth embryogenesis occurs as a result of migration of neural crest cells that specialize in formation of specific type of teeth. Embryogenesis which is controlled by genes could be related to teeth type, shape, affiliation and prolapse with the help of homebox genes such as msh homebox MSX1 and MSX2. ${ }^{8}$

Hypodontia is a multifactorial condition which occurs due to environmental, hereditary, endocrine disturbances and genetic effects. Genes that are responsible for hypodontia are paired box 9 (PAX 9), axis inhibition protein 2 (AXIN2), MSX1 and EDA. Most commonly mutated gene in hypodontia is WNT10A. Environmental factors include presence of cleft palate and the use of chemotherapy in early infancy for childhood malignancy. ${ }^{9}$ Other causes include space limitation, functional abnormalities of the dental epithelium, physical obstruction or disruption of the dental lamina or failure of initiation of the underlying mesenchyme. ${ }^{2}$

Prevalence of hypodontia ranges from 3.2 to $7.6 \%$ in worldwide population with a lowest in Australian population with $1 \%$ and highest in Japanese population with $30 \%{ }^{1,10}$ In Indian population, it has been found around $4.19 \%{ }^{11}$ Mandibular second premolars (2.91$3.22 \%)$ and maxillary lateral incisors (1.55-1.78\%) are commonly involved teeth. ${ }^{2}$ Mandibular lateral incisors absence is a rare finding and usually occurs in Chinese population. ${ }^{12}$ The present case shows bilateral congenital missing of both lower second premolars and lateral incisors which is rare specially in nonsyndromic patient.

Dental features which commonly occur in association with hypodontia are microdontia which may be localized or generalized, conical teeth, ectopic eruption into the space of lateral incisor, retained primary teeth may be infraoccluded, reduced alveolar development, delayed eruption of permanent teeth, increased tendency toward class III malocclusion and reduced lower anterior facial height. ${ }^{6}$ More than 50 syndromes of the head and neck
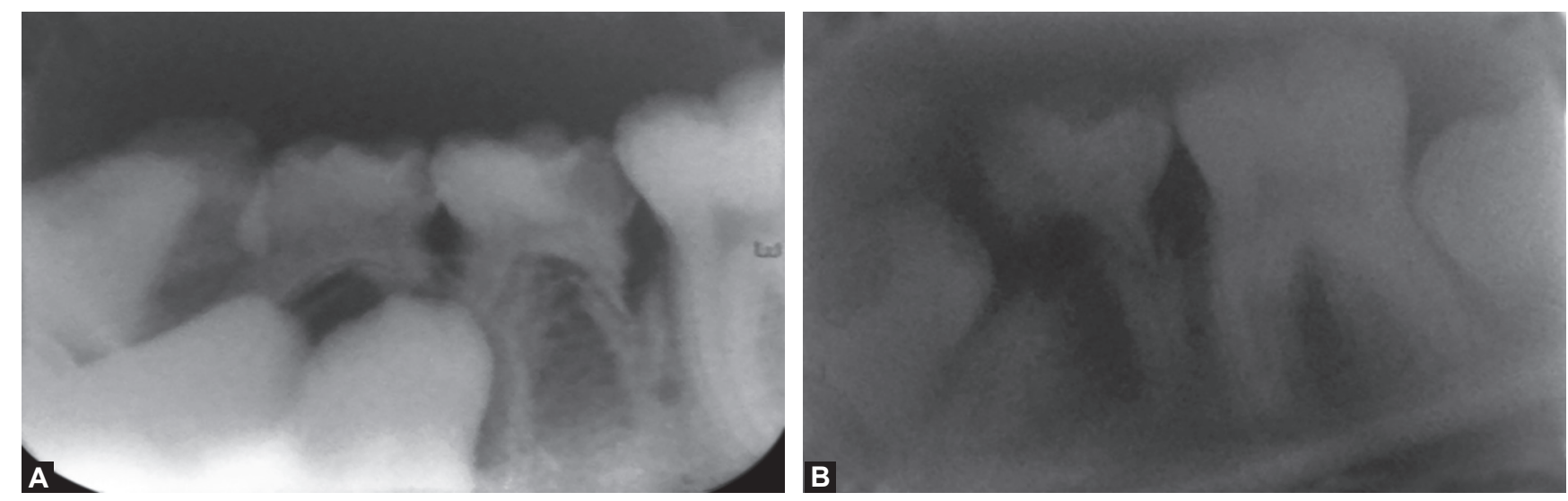

Figs $2 \mathrm{~A}$ and B: Intraoral periapical radiolucency showing deciduous second molar with pulpal involvement and periapical radiolucency 


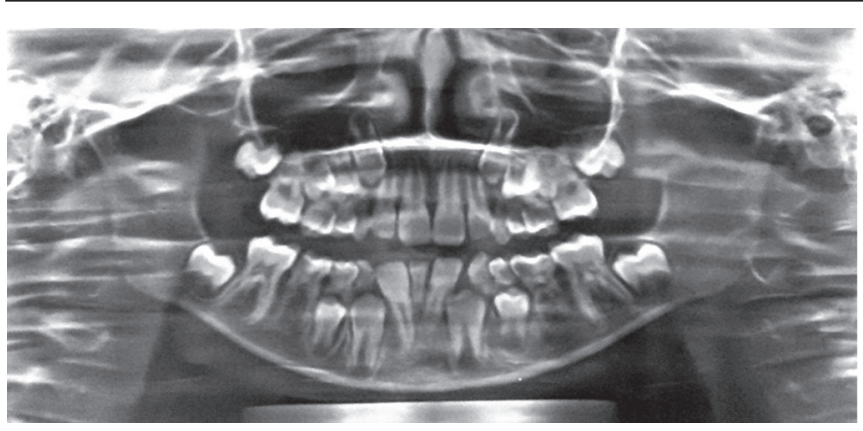

Fig. 3: Orthopantomograph (OPG) showing congenitally missing bilateral mandibular lateral incisors and second premolars

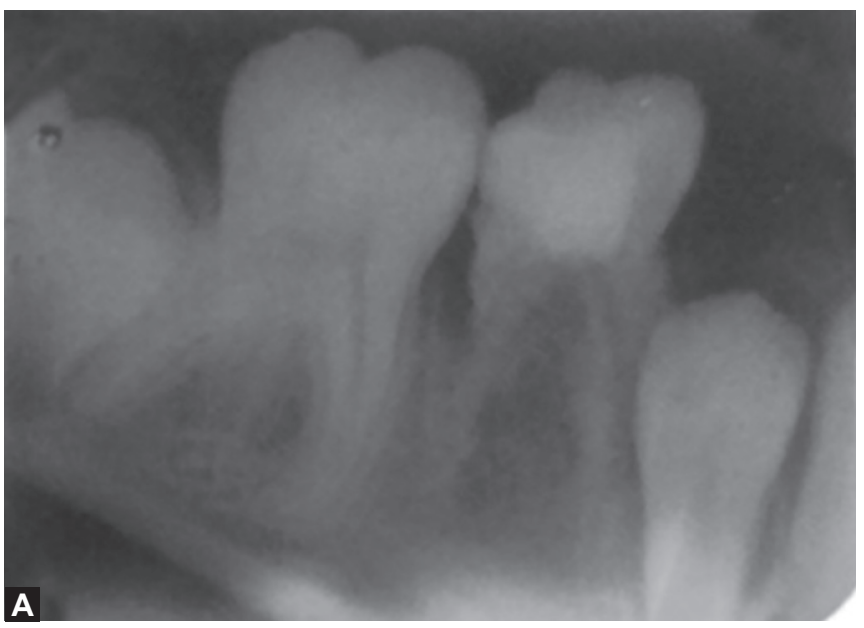

\section{REFERENCES}

1. Polder BJ, Van't Hof MA, Van der Linden FP GM, et al. A metaanalysis of the prevalence of dental agenesis of permanent teeth. Community Dent Oral Epidemiol 2004;32:217-226.

2. Stewart RE, Barber TK, Troutman KC, Wei SHY. Textbook of pediatric dentistry: scientific foundation \& clinical practice. St. Louis: CV Mosby Co 1982;87-109.

3. Park JH, Kim DA, Tai K. Congenitally missing maxillary lateral incisors: treatment. Dent today 2011 May;30(5):84-86.

4. Xuan K, Jin F, Liu YL, Yuan LT, Wen LY, Yang FS, et al. MSX1 gene in Chinese family with autosomal-dominant oligodontia. Arch Oral Biol 2008;53:773-779.

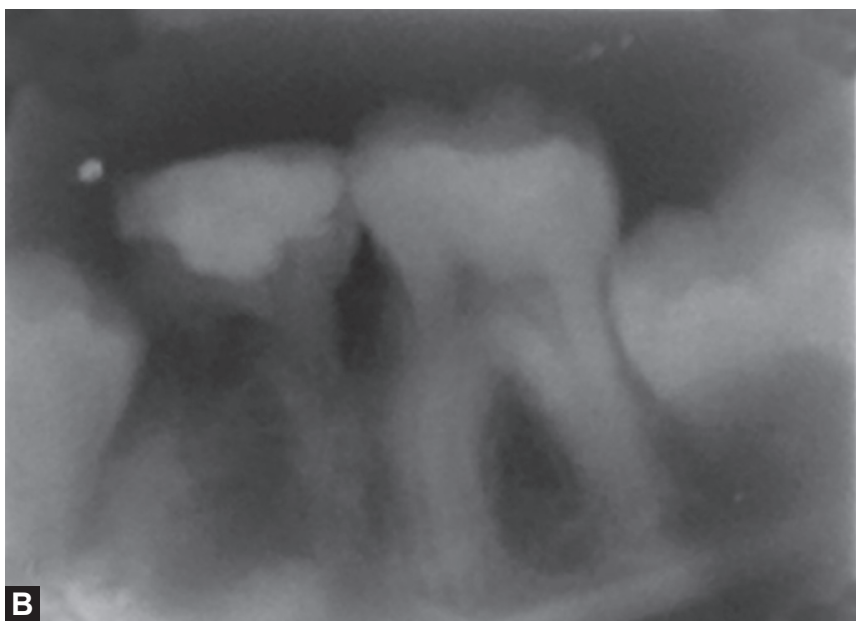

Figs 4A and B: Intraoral periapical radiolucency after 3 weeks showing periapical radiolucency had subsided and permanent first premolars were erupting along the straight pathway of eruption

are reported in literature which occurs in association with hypodontia. Common ones are extradermal dysplasia, van der Woude syndrome, cleft lip and palate, pierre robin syndrome and Down syndrome. ${ }^{13}$

Most patients seek orthodontic treatment to improve their facial esthetic rather than the function. However, periodontal damage, malocclusion, esthetic problems and alveolar bone deficiency are some of the complications following hypodontia. Hence, multidisciplinary approach should be considered while treating a patient with missing premolars and lateral incisor. It may include prosthodontics, orthodontics, esthetic dentistry and implantology. 6

In the present case, child was in mixed dentition stage; hence, a preventive treatment is devised till the orthodontic and prosthodontic treatment to be considered.

\section{CONCLUSION}

Anomalies of teeth involve dentition alterations that can lead to dental disharmony, functional and esthetic alteration. Hence, early diagnosis and proper management of anomalies is necessary.
5. Khalaf K, Miskelly J, Voge E, Macfarlane TV. Prevalence of hypodontia and associated factors: a systematic review and meta-analysis. J Orthod 2014;41:299-316.

6. Gill DS, Barker CS. The multidisciplinary management of hypodontia: a team approach. British Dent J 2015;218(3):143-149.

7. Endo T, Ozoe R, Kubota M, Akiyama M, Shimooka S. A survey of hypodontia in Japanese orthodontic patients. Am J Orthod Dentofacial Orthop 2006;129:29-35.

8. Zhang W, Qu HC, Zhang Y. Association of MSX1 and TGF-beta1 genetic polymorphisms with hypodontia: metaanalysis. Genet Mol Res 2014;13:10007-16.

9. Bohring A, Stamm T, Spaich C, Haase C, Spree K, Hehr $\mathrm{U}$, et al. WNT10A mutations are a frequent cause of a broad spectrum of ectodermal dysplasias with sex-biased manifestation pattern in heterozygotes. Am J Hum Genet 2009;85:97-105.

10. Ibrahim Al, Rafi AT, Mohammed A. A review of hypodontia: classification, prevalence, etiology, associated anomalies, clinical implications and treatment options. World J Dent 2013;4:117-125.

11. Gupta SK, Saxena P, Jain S, Jain D. Prevalence and distribution of selected developmental dental anomalies in an Indian population. J Oral Sci 2011;53:231-238.

12. Fekonja A, Cretnik A, Takac I. Hypodontia prevalence and pattern in women with epithelial ovarian cancer. Angle Orthod 2014;84:810-814.

13. Ranta R. Numeric anomalies of teeth in concomitant hypodontia and hyperdontia. J Craniofacial Genetics Developmental Biol 1988;8:245-251. 